\title{
Death with functioning kidney transplant: an obituarial analysis
}

\author{
Puneet Sood $\cdot$ Yong R. Zhu $\cdot$ Eric P. Cohen
}

Received: 27 May 2009/ Accepted: 18 February 2010/Published online: 3 June 2010

(C) The Author(s) 2010. This article is published with open access at Springerlink.com

\begin{abstract}
Background Death with a functioning kidney graft (DWFG) is now a major cause of graft loss after renal transplantation, occurring in up to $40 \%$ of cases. Its occurrence provides insight into the medical care of subjects with a functioning kidney transplant. In this study, we used the time to DWFG as an endpoint, to test whether improved medical care has contributed to better kidney transplant outcomes.

Methods We used single-center data from the Milwaukee Regional Medical Center and Froedtert Hospital, on kidney-only transplants from 1969 through 2005. A total of 3,157 kidney transplants were done at our center during this time. There were 714 deaths with functioning kidney. We also recorded the major causes of DWFG over the time period from 1969 through 2005 divided into 3 epochs. The data were analyzed as a serial collection of yearly obituaries.
\end{abstract}

\section{P. Sood $(\square)$}

Division of Nephrology, University of California,

521 Parnassus Avenue, Clinical Sciences, Room C443,

San Francisco, CA 94143-0532, USA

e-mail: puneet.sood@ucsf.edu

Y. R. Zhu

Division of Transplant Surgery, Medical College of Wisconsin, Milwaukee, WI, USA

\section{E. P. Cohen}

Nephrology Division, Medical College of Wisconsin, Milwaukee, WI, USA
Results The time to DWFG has increased to 10 years despite a 20 -year increase in the mean age of transplant recipients over the same time period. Conclusions Better pre-transplant evaluation, improved treatments for hypertension and hyperlipidemia, improved management of acute myocardial infarction, superior immunosuppressive protocols and better prophylaxis and treatment of infectious diseases have all likely contributed to this trend.

Keywords Death with functioning graft . Survival - Cardiovascular care .

Infectious disease prophylaxis · Transplantation

\section{Introduction}

Kidney transplant survival has improved in recent years $[1,2]$. This improvement is often credited to better immunosuppressive protocols, with reduced rejection rates and also lesser medication toxicity. But general medical care may also affect kidney transplant patient survival. This aspect of post-transplant care has not been well quantified, and its impact may be ignored if graft survival rates are censored for death with functioning graft. Yet, death with a functioning kidney graft (DWFG) is a major cause of graft loss after renal transplantation, occurring in $10-40 \%$ of transplants [3-6]. Because it does not consider loss of the kidney transplant and resumption of dialysis, time to DWFG does not incorporate graft loss due to 
immunological factors. Rather, time to DWFG may provide insight into post-transplant medical care. In this study, we used the time to DWFG as an endpoint, to test whether improved medical care has contributed to better kidney transplant outcomes.

\section{Methods}

We used single-center data from the Milwaukee Regional Medical Center and Froedtert Hospital, on kidney-only transplants from 1969 through 2005. A total of 3,157 kidney transplants were done at our center during this time. The cause of ESRD was ascertained at the time of first encounter or listing and from the Form CMS 2728. There were 714 deaths with functioning kidney, 564 in subjects with deceased donor transplants. We also recorded the major causes of DWFG over the time period from 1969 through 2005 divided into 3 epochs. The cause of death was ascertained from the hospital records and death certificate information. The data were analyzed as a serial collection of yearly obituaries. That is, the median age at time of death was calculated for all subjects dying in a particular year, and those median ages were graphed as a function of year of death, rather than year of transplant. This method is realistic because it corresponds to day-today experience. It also eliminates confounding by less time of follow-up in more recent years. Finally, we obtained the general population mortality data from the State of Wisconsin for the years included in this study. The study is fully approved by the Human Research Review Committee of the Medical College of Wisconsin.

\section{Results}

In the time period from 1969 to 2005 , there were 714 deaths with functioning kidney, 564 in subjects with deceased donor transplants. Of these 564 subjects, $65 \%$ were men and $35 \%$ were women recipients of deceased donor kidney transplant. Of the subjects with DWFG who had living donor transplants, 64\% were men and $36 \%$ were women. There has been an increase in median time to DWFG from about one year in 1969 to 10 years in year 2005 (Fig. 1). During

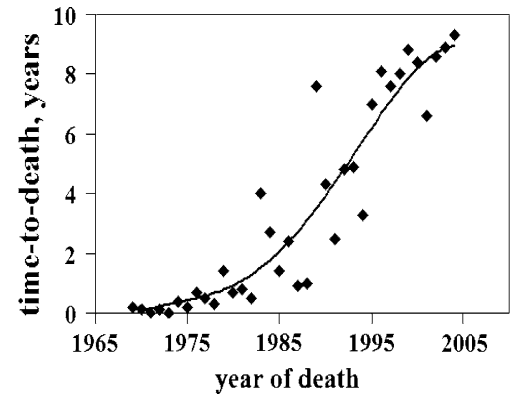

Fig. 1 Showing increasing median time to death with functioning graft (DWFG) from years 1969 through 2005

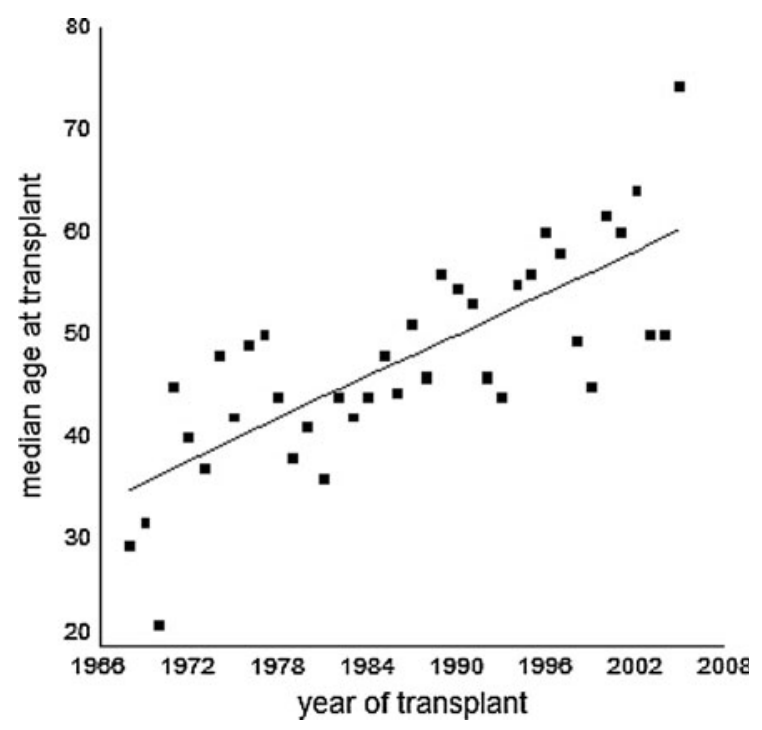

Fig. 2 Showing an increasing median age at time of transplant with the each year of transplant

this same time, there was a 20-year increase in median age of kidney transplant recipients at time of transplant (Fig. 2). In addition, in epoch 1, (19691980), $5 \%$ of the kidney disease was caused by diabetes whereas in epoch 2 (1981-1992) and epoch 3 (1993-2005), respectively, $41 \%$ and $47 \%$ of the kidney disease was secondary to diabetes. For epochs 1,2 and 3, hypertension was the underlying cause of kidney disease in 2\%,11\% and $10 \%$ respectively. The increase in time to DWFG is 10 years over the same total time period. A separate analysis of deaths by epoch showed changes with time, notably a reduced proportion of death caused by infection (Fig. 3). 


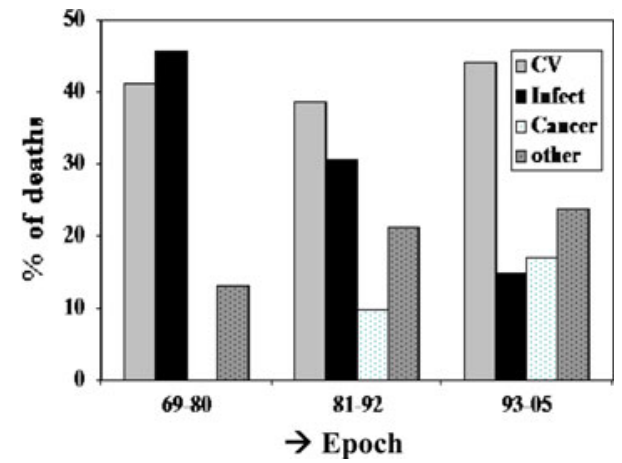

Fig. 3 Depicting changing trends in cause of death kidney transplant recipients in each of the three epochs (1969-1980; 1981-1992; 1993-2005)

\section{Discussion}

These data show an increase in median time to death with functioning graft over the last 30 years despite the aging population of transplant recipients, and in the face of a substantial increase in the numbers of patients with diabetes as the cause of their kidney failure. Over the same time period as this study, the median age at death in general population of the State of Wisconsin for the same period has increased from age 73 to age 80 [7]. It is likely that the subjects of our study were helped by the medical care advances over this time, in a way that is parallel to the general population. A recent review of transplant outcomes in the elderly population by Saxena et al. have also highlighted better survival in elderly transplant recipients attributable to multiple factors [8].

Ojo et al. also showed improvement in survival of subjects with a functioning kidney transplant [6]. But this analysis was limited to the time period 1988 to 1997. Our cohort includes subjects before that time period and after it. In addition, while Ojo et al. reported a decline in DWFG in terms of risk reduction, our analysis additionally provides the immediately useful practical information of the actual number of years of patient survival with a functioning transplant.

Better pre-transplant evaluation, pre- and posttransplant cardiovascular risk factor modification and treatment, and better post-transplant care of infections are likely contributors to the increasing time to death with functioning graft.

Recent reports show a significant reduction in coronary heart disease mortality in the general population in the year 2000 when compared to the year 1980 [9]. This effect is attributable equally to reduction in risk factors and use of evidence-based medical therapies. In the case of kidney transplant patients, the role of hyperlipidemia and other risk factors is similar to that of the general population [10]. There are additional risk factors in subjects with kidney transplants, such as the effect of reduced kidney function, which are not present in the general population. Nonetheless, death from IHD (Ischemic heart disease) in people with a kidney transplant is significantly associated with traditional risk factors of hypertension, increasing age, lower HDL, diabetes mellitus (DM), hypercholesterolemia, baseline congestive heart failure (CHF) and cardiovascular disease (CVD) history [10]. Ivens et al. concluded that the high incidence of CVD following renal transplant was mainly due to increased prevalence and accumulation of classic risk factors before and following transplant [11]. Since the cardiovascular disease in kidney transplant patients shares the same risk factors for cardiovascular disease as in general population, their modification, as observed in the general population, is expected to improve the cardiovascular disease outcomes [12].

Indeed, the European ALERT extension study demonstrated a significant reduction in risk of cardiac death and non-fatal MI in renal transplant patients with moderate elevation in cholesterol treated with fluvastatin when compared to those treated with placebo [13]. An American study reported a $24 \%$ reduction in mortality in patients treated with statins when compared to placebo [14]. More recently, a study looking at a cohort of 2041 first-time renal transplant recipients from 1990 to 2003 reported improved survival in patients on statins when compared to not being on statins (HR (adj) 0.64, 95\% CI 0.48-0.86) [15]. In confirmation of these reports, the USRDS in its 2007 annual data report shows significant improvement in 1- and 5-year survival of kidney transplant patients after a diagnosis of heart disease [16]. In neither the USRDS data nor in the present report can we confirm the role of statins because we have no stored data on their use. It is likely, however, that use of statins has increased steadily since 1985 , which is at or about the time when time to death with functioning transplant began to increase (Fig. 1). Hypertension control appears to play a significant role in better kidney transplant survival. A cohort analysis from the Collaborative 
Transplant Study reported better kidney transplant survival and lesser cardiovascular deaths with control of the systolic blood pressure to less than $140 \mathrm{~mm} \mathrm{Hg}$ [17]. Over the past three decades, control of the blood pressure has become more effective, and with less side effects. Use of angiotensin-converting enzyme inhibitors has increased steadily since the mid-1980s, coinciding with the upswing in time to death with functioning graft (Fig. 1) This aspect of cardiovascular risk reduction is likely to have played an important role in improving kidney transplant patient survival in our cohort. But we cannot confirm this, because we have no stored data on blood pressures of type of antihypertensive use in these patients.

Treatment for disease has also improved. In the general population, there has been a $30 \%$ reduction in one-year mortality after myocardial infarction in 1992-1994 when compared to 1981-1983, which is attributed to evidence-based therapies [18]. Better cardiac interventions over the years have also helped in subjects with functioning kidney transplants. Herzog and colleagues retrospectively examined outcomes of renal transplant recipients hospitalized during 1977-1996 for a first acute myocardial infarction (AMI) and reported markedly improved long-term survival of renal transplant recipients after AMI in the modern treatment era [19].

Acute rejection episodes have been identified as risk factors for development of cardiovascular disease namely ischemic heart disease and cerebral vascular disease [13]. Use of better immunosuppression protocols over the past three decades has thus contributed indirectly to decreased prevalence of CVD mortality by reducing rejection rates. A recent study from Vienna looked at 1823 first kidney transplants from 1990 to 2003. They found significantly better patient survival for patients who received Calcineurin inhibitor containing maintenance immunosuppression when compared to those without it $(0.41,95 \% \mathrm{CI}$ 0.30-0.57) [20].

Besides the decreased cardiovascular mortality, a decrease in infection-related post-transplant mortality has also contributed to better patient survival [5]. Better infection control practices, better antibiotics and anti-virals, and reduced overall immunosuppression are all likely factors in the decrease in infectionrelated mortality in transplant patients. Specifically, valaciclovir prophylaxis has been reported to improve survival in renal transplant patients who are at high risk for developing CMV disease [21]. A recent Cochrane database systematic review confirmed this by examining 34 studies with 3,850 participants. This meta-analysis study concluded that prophylaxis with antiviral medications reduces CMV disease and CMV-related mortality in solid organ transplants [22]. Prophylaxis with acyclovir, ganciclovir or valacylcovir has also been reported to reduce the risks of diseases associated with herpes simplex, herpes zoster, bacterial and protozoal infections [23]. Universal immunization of a transplant recipient has also likely contributed to decreased infectious mortality. Better pre-transplant evaluation of the recipient in terms of cancer screening, cardiovascular risk factors identification and their modification could contribute to this trend of increased survival to DWFG event. However, after transplantation, in subjects with functioning kidney transplants, routine testing for cancer in all likelihood does not reduce overall mortality and thus cannot explain the impressive gains in patient survival to time of DWFG that we have shown [24, 25].

Our 10-year median time to DWFG contrasts with data from USRDS that report a median time to DWFG of 6.5 years in the year 2005. Ours is a singlecenter data, and its values can deviate from the population mean, in this case USRDS data, within 2 standard deviations of the population mean. Another possible explanation is the differential age distribution of our data and the USRDS data such that a relatively younger segment of population was being transplanted during each year when compared to our corresponding single-center experience.

This is consistent with the report of Ojo et al., who found that increasing age at transplant was significantly associated with DWFG [6]. Also, younger patients are more likely to survive to graft failure and resume dialysis.

A major limitation of our study is its retrospective nature. We see an increase in time to death with functioning graft (DWFG) in our data, and we have cited published data to attribute it to improved medical practices, the foremost of which being modification of cardiac risk factors and management of cardiac and infectious disease. However, because of the retrospective nature of our study, which spanned over more than three decades, some of the data on such practices were not systematically collected and stored. In addition, no data were collected and stored on non-traditional risk factors as many of them were identified as novel $\mathrm{CV}$ 
risk factors only in the most recent epoch. Thus, although we see benefit in terms of improved outcomes at our center, the current study is somewhat limited in attributing those benefits directly to our changing and improved practices. Nevertheless, the medical practices that led to the improvement in time to DWFG and their approximate time of introduction into clinical practice are similar to the adoption of those practices at out center.

In conclusion, patient survival with a functioning kidney transplant has increased. This points to improvements in medical care as important factors in better kidney transplant outcomes.

Factors that may play a role in this improvement include: better pre-transplant evaluation, better treatment for traditional cardiovascular risk factors such as hypertension and hyperlipidemia, improved management of myocardial infarction, superior immunosuppressant protocols and better prophylaxis and treatment of infections. The advances in medical care that have helped the general population to live longer are likely to have also improved the survival of kidney transplant patients.

Open Access This article is distributed under the terms of the Creative Commons Attribution Noncommercial License which permits any noncommercial use, distribution, and reproduction in any medium, provided the original author(s) and source are credited.

\section{References}

1. Morris PJ, Johnson RJ, Fuggie SV et al (1999) Analysis of factors that affect outcome of primary cadaveric renal transplantation in the UK. Lancet 354(9185):1147-1152

2. Hariharan S, Johnson CP, Bresnahan BA, Taranto SE, Stablein D (2000) Improved graft survival after renal transplantation in the United States, 1988 to 1996. N Engl J Med 342(9):605-612

3. Shimmura H, Tanabe K, Tokumoto T, Ishida H, Ishikawa N, Miyamoto N, Shirakawa H, Toma H (2004) Analysis of cause of death with a functioning graft: a single center experience. Transplant Proc 36(7):2026-2029

4. El-Agroudy AE, Bakr MA, Shehab EL, Dein AB, Ghoneim MA (2003) Death with functioning graft in living donor kidney transplantation: analysis of risk factors. Am J Nephrol 23(3):186-193

5. Matas AJ, Humar A, Gillingham KJ et al (2002) Five preventable causes of kidney graft loss in the 1990s: a single center analysis. Kidney Int 62(2):704-714

6. Ojo AO, Hanson JA, Wolfe RA et al (2000) Long term survival in renal transplant recipients with graft function. Kidney Int 57 (1):307-313
7. http://dhfs.wisconsin.gov/deaths/index

8. Saxena R, Yu X, Giraldo M, Arenas J, Vazquez M, Lu CY, Vaziri ND, Silva FG, Zhou XJ (2009) Renal transplantation in elderly. Int Urol Nephrol 41(1):185-210

9. Ford ES, Ajani UA, Croft JB, Critchley JA, Labarathe DR, Kotke TE, Giles WH, Capewell S (2007) Explaining the decrease in US deaths from coronary disease, 1980-2000. N Engl J Med 356(23):2388-2398

10. Aakhus S, Dahl K, Wideroe TE (2004) Cardiovascular disease in stable renal transplant patients in Norway: morbidity and mortality during a 5-years follow up. Clin Transplant 18(5):596-604

11. Ivens K, Aker S, Grabensee B, Heering P (1999) Incidence of Cardiovascular risk factors and complications after kidney transplantation. Med Klin (Munich) 94(9):478-484

12. Djamali A, Samaniego M, Muth B et al (2006) Medical care of kidney transplant recipients after the first posttransplant year. Clin J Am Soc Nephrol 1(4):623-640

13. Holdaas H, Fellstrom B, Cole E, Nyberg G, Olssan AG, Pettersen TR, Madsen S, Gronhaggen-Riska C, Neumayer HH, Jardine AG (2005) Long-term cardiac outcomes in renal transplant recipients receiving fluvastatin: the ALERT extension study. Am J Transplant 5(12):2929-2936

14. Cosio FG, Pesavento TE, Pelletier RP, Henry M, Ferguson RM, Kim S, Lemeshow S (2002) Patient survival after renal transplantation. III: The effects of statins. Am J Kidney Dis 40(3):638-643

15. Wiesbauer F, Heinz Georg, Mitterbauer C, Harnoncourt F, Horl WH, Oberbauer R Statin use is associated with prolonged survival of renal transplant recipients. J Am Soc Nephrol 19:211-2218

16. http://www.usrds.org/2007/pdf/06_hosp_morte_07.pdf

17. Opelz G, Dohler B (2005) Improved long-term outcomes after renal transplantation associated with blood pressure control. Am J Transplant 5(11):2725-2731

18. Gottlieb S, Goldbourt U, Boyko V, Harpaz D, Mandelzweig L, Khouty Z, Stern S, Behar S (2000) Mortality trends in men and women with acute myocardial infarction in coronary care units in Israel. A comparison between 1981-1983 and 1992-1994. Eur Heart J 21(4):284-295

19. Herzog CA, Ma JZ, Collins AJ (2000) Long term survival of renal transplant recipients in the United States after acute myocardial infarction. Am J Kid Dis 36(1):145-152

20. Heinze G, Oberbauer R, Kainz A, Mitterbauer C, Koppelstaetter C, Hörl WH, Mayer G (2009) Calcineutrin inhibitor based immunosuppressive therapy, donor age and long term outcome after kidney transplant. Transplantation 87(12):1821-1829

21. Tilden DP, Chapman J, Davey PJ, Solly ML, Crowley S (2004) A decision-analytic economic evaluation of valaciclovir prophylaxis for the prevention of cytomegalovirus infection and disease in renal transplantation. Clin Transplant 18(3):312-320

22. Hodson EM, Craig JC, Strippoli GF, Webster AC (2008) Antiviral medications for preventing cytomegalovirus disease in solid organ transplant recipients. Cochrane Database Syst Rev 16(2):CD003774

23. Hodson EM, Jones CA, Webster AC, Strippoli GF, Barclay PG, Kable K, Vimalachandra D, Craig JC (2005) Antiviral medications to prevent cytomegalovirus disease and early death in recipients of solid-organ transplants: a systematic 
review of randomized controlled trials. Lancet 365(9477): 2105-2115

24. Kiberd BA, Keough-Ryan T, Clase CM (2003) Screening for prostate, breast and colorectal cancer in renal transplant recipients. Am J Transplant 3(5):619-625
25. Wong G, Chapman JR, Craig JC (2008) Cancer screening in renal transplant recipients: what is the evidence? Clin J Am Soc Nephrol 3(Suppl 2):S87-S100 